Monatsschrift für Geburtshülfe u. Gynäkologie 1904;20:251-252

\title{
Naturforscher-Versammlung zu Breslau
}

18.-24. September 1904.

19. Abteilung: Geburtshilfe und Gynäkologie.

Einführende: Geh. Medizinalrat Prof. Dr. Küstner, Prof. Dr. Fränke1'

Direktor Dr. Baumm, Primärarzt Dr. Asch.

Schriftlührer: Dr. L. Fränkel, Privatdozent Dr. Dienst.

Sitzungsraum: Königl. Frauenklinik, Maxstr. 3.

1. Ascb-Breslau:

Schnittführung und Naht.

Diagnose und Behandlung der Ehegonorrhoe.

Ghrobak- $\Lambda$ Vien: Thema vorbehalten.

Deli us-Hannover: Der Einûuss cerebraler Momente auf die Menstruf ${ }^{\circ} u$ und die Behandlung von Menstruationsstörungen durch hypn $\theta$ tısC Suggestion.

4 Dienst-Breslau: Demonstration von Präparaten.

ò. Do der I ein-Tubingen: Abdominelle Exstirpation des carcinoroatosen Uterus.

Fellner-Wien: Thema vorbehalten.

Franqué- Prag: Über Hinterscheitelbeineinstellung.

W. A. Freund-Berlin: Über die neuesten Ausgestaltung en der abdomt-nalen Totalexstirpation des carcinomatösen Uterus im Anschluss aD c Vorstellung einer im Juni 1878 operierten Frau. H. Freund-Strassburg: Thema vorbehalten.

Fritsch-Bonn: Operative Behandlung der Retrodeviationen des Oterus.

v. Guérard-Düsseldorf: Kritische Bemerkuagen über den Wert des $\mathrm{f}^{1} / 8$ ser-schnittes bei

Eklampsie.

Hahn-Wien: Zur Therapie der Dysmennorrhoe.

Halban-Wien: Thema vorbehalten.

von Herff-Basel: Über die Zangenanlegung bei Hinter3cheitelbem einstellung.

Hitschmann-Wien: Thema vorbehalten.

Jung-Greifswald: Anatomische Untersuchungen über den Nerven»pPara ' der weiblichen

Genitalien.

Krönig-Freiburg: Thema vorbehalten.

Küstner-Breslau: Zur Kritik der Methodik aseptischer Kauteleo auí dem Gebiete der operativen Gynäkologie.

Ferd. H. Ku m pf-Karlsbad:

Über die Thure-Brandt sche Methode,

Demonstration von Elektrothermen.

Le op old-Dresden: Thema vorbehalten. 
Martin-Greifswald: Beitrag zur Lumbalanaesthesie Gebärender.

Merttens-Düsseldorf: Extrauteringravidität und Deeiduom.

Müller-Bern: Thema vorbehalten.

Nenadovics-Franzensbad: Zur Balneotherapie der Frauenkrank"el

25. Neugebauer-Warschau: Thema vorbehalten.

2(5. Opitz-Marburg: Schwangerschaft und Pyelitis.

27. Pfannenstiel-Giessen: Über die Behandlung der chronischen $1 / 8$ nct0" metritis.

252

Personalien und Tagesnachrichten.

28. Polano-Würzburg:

Der Antitoxiniibergang von der Mutter auf das Kind, ein Beitrag zur Physiologie der Placenta.

Demonstration von Präparaten.

Poten-Hannover: Thema vorbehalten.

Schatz-Rostock: Die instrumentelle Eröffnung des schwangeren und des nicht schwangeren Uterus.

Schaeffer-Heidelberg:

TJber Wehenmechanismus.

Über Endometritis circumscripta.

Schauta-Wien: Thema vorbehalten.

Sellheim-Freiburg: Zur Mechanik der Geburt.

Tuszkai-Marieabad: Physikalische Heilbestrebungen in der Gynäkologie.

Veit-Halle: Thema vorbehalten.

Werth-Kiel: Mitteilungen zur Technik und aseptischen Gestaltung der Laparotomie.

$\Lambda$ Vertheim-Wieu: Thema vorbehalten.

von Winckel-Miinchen: Thema vorbehalten.

Winter-Königsberg:

Tuberkulose und Schwangerschaft,

Zur Patbologie des Uterus rudimentarius.

Vorzustellende Kranke finden in der Königl. Universitäts-Frauenklinik

Aufnahme.

Die Abteilung ladet ein: die Abteilung 3 (Angewandte Mathematik und Physik) und 14

(Anatomie und Physiologie) zu: Sellheim-Freiburg: Zur Mechanik der Geburt;

die Abteilung 21 (Neurologie und Psychiatric) zu: Delius-Hannover: Der Einfluss cerebraler

Momente auf die Menstruation und die Behandlung von Menstruationsstörungen durch hypnotische Suggestion;

die Abteilung 25 (Dermatologie u. s. w.) zu: Asch-Breslau: Diagnose und Behandlung der

Ehegonorrhoe.

Personalien und Tagesnachrichten.

D0p XV. internat. medizin. Kongress findet vom 19.-26. April 1906 in Lissabon statt.

Generalsekretär ist Prof. Miguel Bombarda. Für die Gynäkologische Sektion sind folgende

Diskussionsthemata aufgestellt:

Behandlung der Retrodeviationen des Uterus. 
Behandlung der Uterusmyome.

Tuberkulose der Adnexorgane.

Herr von Ott wird den Internationalen Gynäkologenkongress auf den 12. September 1905 nach Petersburg einladen.

In Parma habililierte sich Dr. E. Alfieri für Geburtshülfe und Gynäkologie. 